\title{
OPEN Optimisation of T2 and T2* sequences in MRI for better quantification of iron on transfused dependent sickle cell patients
}

\author{
Azza Ahmed ${ }^{1 凶}$, Amani Baldo ${ }^{2}$, A. Sulieman ${ }^{3}$, Hind Mirghani², Fouad A. Abolaban ${ }^{4}$, \\ I. I. Suliman ${ }^{1,5}$ \& Isam Salih ${ }^{1,6}$
}

This work aimed to investigate the effect of different shim techniques, voxel sizes, and repetition time (TR) on using the T2 and T2* sequences to determine their optimum settings to investigate the quantification of iron in transfused dependent sickle cell patients. The effect of each of these parameters was investigated on phantoms of different Gadolinium (Gd) concentrations, on 10 volunteers and 25 patients using a1 5T MRI Philips scanner. No significant difference between the three shim techniques was noticed in either T2 or T2* sequence measurements. Pixel sizes of $1 \times 1$ and $2 \times 2 \mathrm{~mm}$ provided optimum results for T2 measurements. At $1 \times 1 \mathrm{~mm}$ pixel size the T2* measurements experienced less error in measurements than the size of $2.5 \times 2.5 \mathrm{~mm}$ used in the literature. Even though the slice thickness variation did not provide any changes in T2 measurements, the $12 \mathrm{~mm}$ provided optimum T2* measurements. TR variation did not yield significant changes on either T2 or $\mathrm{T} 2$ * measurements. These results indicate that both $\mathrm{T} 2$ and $\mathrm{T} 2$ * sequences can be further improved by providing more reliable measurements and reducing acquisition time.

Sickle cell anaemia (SCA) is a haemoglobinopathy caused from a single point mutation in the $\beta$-chain of human haemoglobin ${ }^{1}$ It causes rigid and sickle-shaped red blood cells, eventually ends up in anaemia, which leads to multiple complications, the most serious of which is the Overt stroke ${ }^{2}$.

In Africa, sickle cell disease is raising great health concerns, particularly in the western part of Sudan among the Baggara (Misseriya tribe). Archibald ${ }^{3}$ stated that the first incidence of sickle cell in Sudan was reported in 1926. The number of cases of this disease reached 30\% among pastoral tribes in western Sudan. Up to $16 \%$ was found in immigrant tribes in the area ${ }^{4}$.

The prevalence of patients with SCA in Sub-Saharan Africa ranges between 5 and $40 \%{ }^{5}$. Their life expectancy is $<20$ years old ${ }^{6}$, and patients younger than 5 years are at high risk of death.

Blood transfusions in sickle cell patients could lead to iron overload particularly in the liver. Iron overload promotes the formation of toxic oxygen radicals that can cause cell damage. Therefore, the knowledge of iron levels in these patients is important to mitigate the health consequences of iron overload.

The levels of iron in blood-transfusion patients are often monitored using the serum ferritin method. This method known to be an unreliable marker for assessing iron in organs such as the heart and liver ${ }^{7}$.

The use of magnetic resonance imaging (MRI) techniques involving transverse relaxation times such as the T2 and $\mathrm{T} 2{ }^{\star}$ have shown promising results in estimating iron concentration in transfusion-dependent patients ${ }^{8,9}$. It is therefore, expected that the serum ferritin and invasive biopsy methods will be replaced by the MRI methods, however, there is limited data in the literature that systematically compared $\mathrm{T} 2$ and $\mathrm{T} 2{ }^{\star}$ sequences in sickle cell anemia (SCA) ${ }^{10}$. For the quantification of iron using MRI techniques, the literature highlighted that MR signal measurements could be affected by sequence protocol ${ }^{11-14}$. Nevertheless, many of these studies were limited to narrow investigations of parameter effects on T2 and T2* measurements. Researchers stated that $\mathrm{T} 2$ techniques

\footnotetext{
${ }^{1}$ Sudan Atomic Energy Commission, Al Gamah Street, P. O. Box 3001, Khartoum, Sudan. ${ }^{2}$ Jaffar Bin Auf Paediatric Hospital, Hosptials Road, Khartoum, Sudan. ${ }^{3}$ Radiology and Medical Imaging Department, College of Applied Medical Sciences, Prince Sattam Bin Abdulaziz University, P. O. Box 422, Alkharj 11942, Saudi Arabia. ${ }^{4}$ Nuclear Engineering Department, Faculty of Engineering, King Abdulaziz University, P. O. Box 80221, Jeddah 21589, Saudi Arabia. ${ }^{5}$ Physics Department, College of Science, Imam Mohammad Ibn Saud Islamic University (IMSIU), P. O. Box 11642, Riyadh, Saudi Arabia. ${ }^{6}$ Basic Science Department, Prince Sattam Bin Abdulaziz University, P. O. Box 422, Alkharj 11942, Saudi Arabia. ${ }^{\square}$ email: azzasabo@hotmail.com; isamsalih@gmail.com
} 
are not affected by the size or shape of the imaging voxel ${ }^{15}$ or shimming techniques ${ }^{16}$, and commented that $\mathrm{T}^{*}$ methods were affected by voxel size ${ }^{17}$, shimming technique and TR.

The empirical effects of shimming techniques, range of voxel sizes, and TRs were not been sufficiently investigated. There is also a lack of understanding of how these changes in parameters affect $\mathrm{T} 2$ or $\mathrm{T} 2{ }^{\star}$ measurements. Reported T2 and T2* values in healthy subjects showed large variation between centres which could be explained due to the acquisition standardisation ${ }^{18}$. While the literature highlighted some guidelines for use of protocols for $\mathrm{T}^{*}$ measurements for cardiovascular image ${ }^{19}$, different acquisition approaches were implemented for $\mathrm{T} 2$ sequences and hence lead to variations in $\mathrm{T} 2$ values $^{20}$. It was also suggested that changing $\mathrm{T} 2^{\star}$ sequence parameters may particularly be significant at high liver concentrations 9 .

Gradient echo sequence $\left(\mathrm{T} 2^{\star}\right)$, and Ferriscan (T2 sequences) have been used for evaluating iron in patients with iron overload, however, Ferriscan is not widely available in Africa.

The T2 sequence is currently a validated sequence for quantification of iron, on the other hand it is technically difficult to implement and requires additional costs and delay for data analysis ${ }^{21}$. The T2 sequence that is available in most scanners is the Fast Spin Echo Sequence or Turbo Spin Echo (TSE) sequence, and has been suggested to be used for iron quantification in transfusion-dependent patients ${ }^{22}$.

Herein we thought to investigate the effect of different shim techniques, voxel sizes, and repetition times (TR) on the quantification of iron in transfusion-dependent sickle cell patients and examining optimum settings for appropriate assessment of patients.

The present study was built on the experimental hypothesis in changing settings and optimizing parameters that may lead to improving the quality of the images produced, which leads to the appropriate evaluation transfusion dependent patients.

\section{Materials and method}

The study was conducted at Dar-Alateeba Hospital, a private hospital located in Khartoum State, Sudan. The measurements involved the use of a $1.5 \mathrm{~T}$ whole-body Philips-MRI scanner. The scanner was equipped with high performance gradients with a maximum strength of $40 \mathrm{mT} / \mathrm{m}$ and a maximum slew rate of $200 \mathrm{~T} / \mathrm{m} / \mathrm{s}$. A four-element torso phased array coil was used for all the scans. The research work explored the best settings that can be used in patients' measurement via investigations conducted first on a phantom, on healthy volunteers and finally on patients as described in the following subsections.

Phantom study and calibration. Phantom was used in the study to simulate the relaxation properties of tissues with different concentrations of iron. It consisted of a Plexiglas holder containing eight plastic bottles, each with a volume of $17 \mathrm{ml}$. Seven bottles contain gadolinium (Gd) at concentration of $0.5,1,2,3,4,5$ and $20 \%$. One of the bottles contained pure water for reference. The effect of the paramagnetic gadolinium on the signal intensity measurements using $\mathrm{T} 1, \mathrm{~T} 2$ and $\mathrm{T} 2{ }^{*}$ sequences has been previously investigated in number of studies $^{23-25}$. Researchers reported that the increase in gadolinium concentrations led to a significant decrease on the $\mathrm{T} 2$ and $\mathrm{T} 2 *$ relaxation ${ }^{26,27}$.

The phantom was initially scanned with a balance fast field echo (FFE) sequence to localise the best plane for analysis. Following this a Turbo Spin Echo (or T2 sequences) and a Gradient Echo (or T2* sequence) were run on the phantom first for calibration using the following parameters: shim $=$ default, pixel size $=2 \times 2 \mathrm{~mm}$, slice thickness $=10 \mathrm{~mm}, \mathrm{TE} / \mathrm{ES}=4.4 / 4.4$ and $\mathrm{TR}=400 \mathrm{~ms}$, which gives an acquisition time of $27.2 \mathrm{~s}$. For T2* sequence the settings were: shim $=$ default, pixel size $=2 \times 2 \mathrm{~mm}$, slice thickness $=10 \mathrm{~mm}, \mathrm{TE} / \mathrm{ES}=2.5 / 1.1 \mathrm{~ms}$, and $\mathrm{TR}=200 \mathrm{~ms}$ and this gives an acquisition time of $7.8 \mathrm{~s}$. The number of echoes for each of the T2 and T2* sequences were 20 and 15 respectively. The scan for each of the two calibration protocols was repeated 3 times. Their mean and standard deviation (SD) of the repeat measurements were obtained.

Following this, optimization protocols were carried out for both the T2 and $\mathrm{T} 2{ }^{\star}$ sequences and it included the effects of the calibration parameters as well as the effects of the remaining: the shim techniques, four pixel sizes, two slice thicknesses and four TR values. For each of the imaging parameters, the scan is repeated three times on which also the mean and SD were calculated for the two sequences.

Volunteers and patients. The results obtained from the phantom study were tested on 10 volunteers and 25 patients. The volunteers were healthy, with no known previous diseases. Initially, serum ferritin measurements were performed for all patients and volunteers. The studied subjects were scanned for the following parameters: Shim default, auto and volume for both T2 and T2* sequence. Pixel sizes were $2 \times 2,2.5 \times 2.5,3.28 \times 3.28$ and $4 \times 4 \mathrm{~mm}$ for T2 and $1 \times 1,2 \times 2,2.5 \times 2.5$ and $3.28 \times 3.28 \mathrm{~mm}$ for T2*. TR values were $245,330,400$, and $500 \mathrm{~ms}$ for T2, and 100,160, 200, and $230 \mathrm{~ms}$ for T2* sequence. Slice thickness of 8,10 , and $12 \mathrm{~mm}$ was also investigated on the phantom for both $\mathrm{T} 2$ and $\mathrm{T} 2{ }^{*}$ sequences.

The patients' study was conducted at the optimum settings obtained from both the phantom and volunteers' studies using both $\mathrm{T} 2$ and $\mathrm{T} 2{ }^{*}$ sequences. These were then compared to the calibration/standard settings, which was implemented before the incremental changes. The settings for the optimised sequence for T 2 were: shim $=$ auto, pixel size $=2 \times 2 \mathrm{~mm}$, slice thickness $=10 \mathrm{~mm}, \mathrm{TE} / \mathrm{ES}=4.4 / 4.4 \mathrm{~ms}$, and TR $=245 \mathrm{~ms}$, with an acquisition time $=16.9 \mathrm{~s}$. The optimised settings for the $2^{*}$ sequence were as follow: shim $=$ default, pixel size $=1 \times 1 \mathrm{~mm}$, slice thickness $=12 \mathrm{~mm}, \mathrm{TE} / \mathrm{ES}=3.7 / 1.7 \mathrm{~ms}$ and $\mathrm{TR}=100 \mathrm{~ms}$-with acquisition time $=5.8 \mathrm{~s}$.

For paediatric patients not able to breath-hold, the sequence was implemented during free breathing using respiratory triggering.

All images acquired were analyzed by plotting a circular region of interest (ROI) using Matlab code (version $2015 b$ ). For the phantom study, the ROI was chosen around each of the eight bottles. As for the volunteers and patients, regions away from the vascular structure were chosen to avoid the contribution of artefacts from the 
blood. The pixel intensities of each of the selected region were averaged together and this region is propagated to other images with longer echo times (TE). The signals were then plotted versus their corresponding TE values using Levenberg-Marquardt algorithm of non-linear curve fitting. Data from T2 and T2* were fitted using a simple exponential decay formula ${ }^{28}$ (Eq. 1):

$$
\mathrm{S}=\mathrm{S}_{0} \exp ^{-\mathrm{TE} / \mathrm{T} 2^{*}}
$$

where $\mathrm{S}$ is the final signal intensity, $\mathrm{S}_{0}$ is the initial signal and TE is the echo time.

All data points at noise level were removed using the truncation method as they are currently widely used ${ }^{29}$ and proven to provide more accurate measurements than the offset and baseline subtraction methods ${ }^{9}$.

All Statistical analysis was performed using EXCEL spreadsheets. To evaluate the differences between the two data sets, a paired Student's $t$-test was calculated. A $p$ value less than 0.05 was considered to be significant. Image analysis were carried out using a personally written programming code (Matlab 2015b).

Ethical approval was obtained from the Health Research Council-National Research Ethics Committee (Ministry of Health, Sudan). The committee was established to review ethical issues in health research proposals submitted for implementation, in the country, and review its results in accordance with regulations that adopt international guidelines. Written informed consent was obtained from each individual for carrying out the studies.

\section{Results and discussion}

Results. The effects of tested parameters were studied in terms of three performance indices: (i) The exponential decay curves of both T2 and T2*; (ii) the magnitude measurements of T2 or T2* values using Eq. 1 and (iii) the correlation coefficient $\mathrm{R}^{2}$, which determines the associated strength between two variables. All results are presented in in Figs. 1, 2, 3, 4 and 5 and Tables 1 and 2.

Phantom and volunteers results. The results of the phantom and volunteers measurements, are presented in Tables 1 and 2 and in Figs. 1 and 2. The figures show plots of the 2\% Gd concentration versus echo time (TE) for three shims, five different pixel sizes, three slice thickness and five TR values. The tables show mean magnitude measurements of Gd concentrations $1-20 \%$ and of 10 volunteers respectively, for both T2 and T2*, using the tested parameters.

Patients results. The results of serum ferritin for the volunteers ranged between 5.5 and $86.8 \mathrm{ng} / \mathrm{ml}$. For the patients, the serum measurements ranged between 179 and $2000 \mathrm{ng} / \mathrm{ml}$. T2 decay curves of standard (TR $400 \mathrm{~ms}$ ) versus optimised (TR $245 \mathrm{~ms}$ ) of a sickle cell patient with iron overload are shown in Fig. 3a. Figure 3b shows the decay curves of the standard $\left(\mathrm{TR}=200 \mathrm{~ms}\right.$ ) versus optimised $(\mathrm{TR}=100 \mathrm{~ms})$ for $\mathrm{T} 2^{\star}$ for the same patient. The magnitude measurements of the 25 patients using the conventional $\mathrm{T} 2$ and $\mathrm{T} 2{ }^{\star}$ sequences versus the new optimised sequences are shown in Fig. 4.

The mean magnitudes of the standard and optimised sequences for T2 were $41.72 \pm 11.82 \mathrm{~ms}$ and $41.10 \pm 11.31 \mathrm{~ms}$, and for $\mathrm{T} 2 *$ they were $5.11 \pm 3.39$ and $5.25 \pm 3.49 \mathrm{~ms}$ respectively.

Figure 5A,B present typical images of a patient acquired using standard and optimised T2*, while Fig. 5C,D obtained using standard and optimised T2, respectively.

\section{Discussion}

It was observed that all decay curves of the phantom studies appeared with offset at the first two data points of the curve. The $\mathrm{T} 2^{\star}$ curves experience slight oscillation. The presence of field inhomogeneities probably caused this oscillation. In Fig. 1 the three shim techniques of $\mathrm{T} 2$ and $\mathrm{T} 2{ }^{\star}$ sequences appeared overlapping, indicating that there is no significant variation between their measurements. Increasing the pixel size (in- resolution) caused an increase in the bandwidth (the scanner was not set for a fixed $\mathrm{Hz} /$ pixel value). Nevertheless, with the increase in bandwidth, the signal-to-noise ratio was reduced simultaneously. The oscillation of data points around the curve was noticed to increase with higher Gd concentrations. Slice thickness had a minor effect on the T2 measures, while the signal intensity was noticed to increase with slice thickness for $\mathrm{T} 2{ }^{\star}$. TR was observed to have a minor effect on both sequence measurements.

Results for volunteers, as shown in Fig. 2, indicate that T2 and T2* were loosely fit to its associated decay curves. Factors such as blood, the anatomy of the organ may have contributed to poor fit. However, T2 curves are bound together, indicating that shim has no significant effect on the measurements. Concurrently, $\mathrm{T} 2{ }^{\star}$ data points exhibited higher oscillation than those of the T2, which is attributed to the presence of field inhomogeneities. Such small oscillation can likely have profound effects on estimating long $\mathrm{T} 2^{\star}$ values ${ }^{30}$. The scattering of data points were noticed to be specifically higher at pixel sizes of $3.28 \times 3.28$ and $4 \times 4 \mathrm{~mm}$, especially for T2* measurements. The trend of the decay curves for the different slice thickness for T2 appeared the same. However, the signal intensity measurements for $\mathrm{T} 2{ }^{*}$ were noticed to be the highest at $12 \mathrm{~mm}$.

Tables 1, 2, 3 and 4 represent the magnitude measurements (with SD) of Gd concentrations of 1-20\% for the different shim techniques, pixel sizes, slice thicknesses, and TR values for both T2 and T2* measurements. Generally, it can be concluded that there is no significant differences in measurements for the three shim techniques. Statistical test showed correlations coefficients $\left(\mathrm{R}^{2}\right)$ ranging from 0.98 to 0.99 for the three shim techniques. However, the $1 \% \mathrm{Gd}$ concentration experienced different $\mathrm{T} 2{ }^{*}$ measurements since it recorded the highest magnitude measurement with the lowest (SD). On the other hand, the increase in pixel sizes resulted in a steady increase in magnitude measurements. The $20 \%$ concentration recorded the lowest $\mathrm{R}^{2}$ and highest SD, especially at smaller pixel sizes. This is probably because low T2 measurements cannot be measured accurately due to the limitation of the short echo time and echo spacing used $(5.9 / 5.9 \mathrm{~ms})$. For $\mathrm{T} 2{ }^{*}$ measurements, the $1 \times 1 \mathrm{~mm}$ recorded the 
(A)

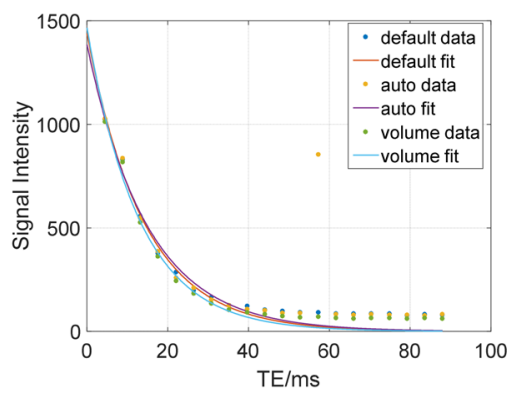

(C)

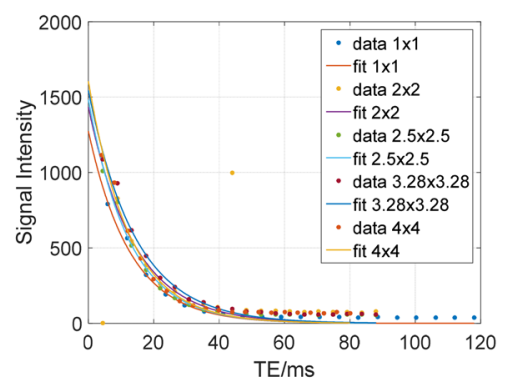

(E)

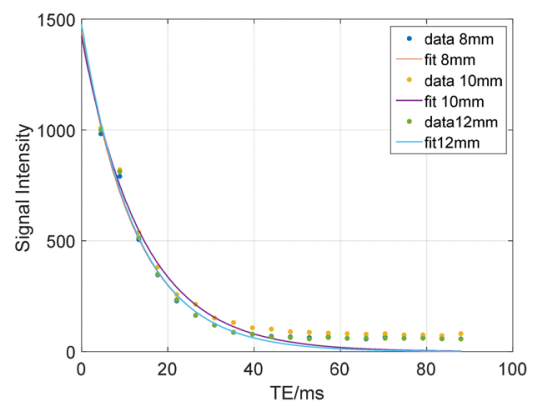

(G)

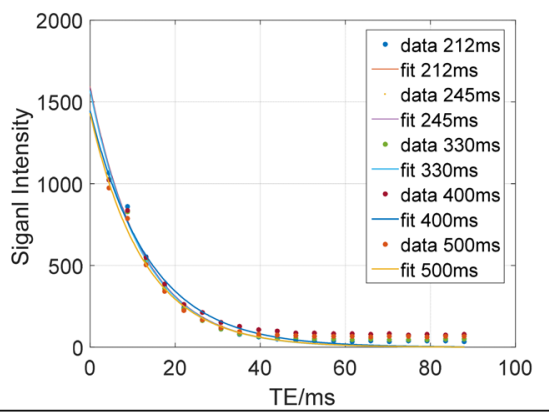

(B)

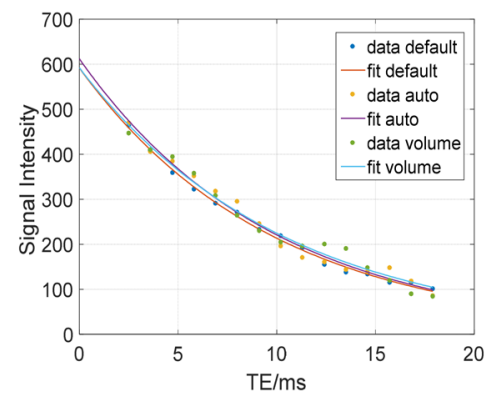

(D)

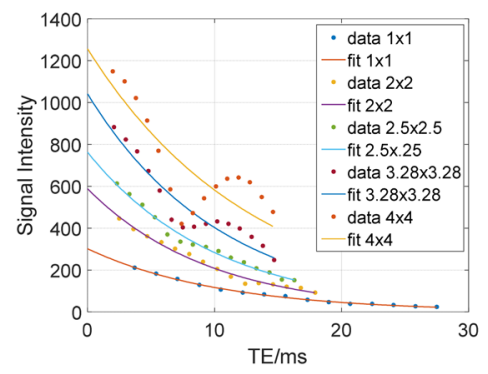

(F)

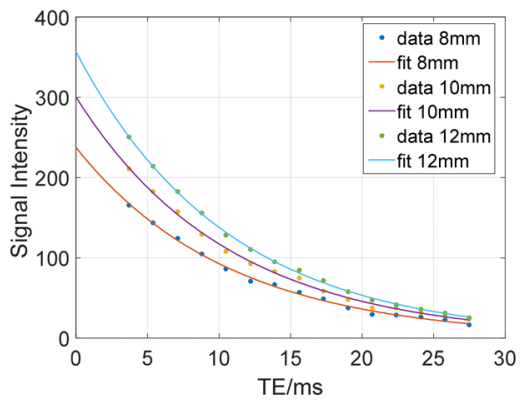

(H)

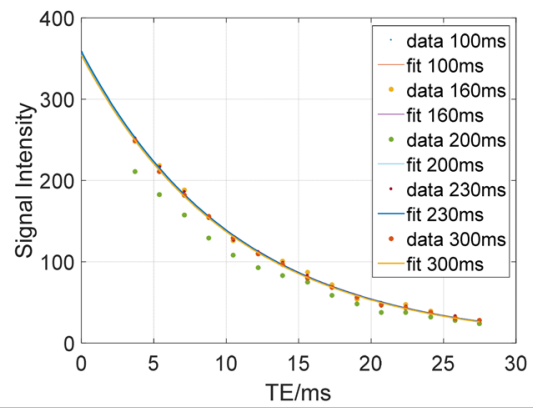

Figure 1. Decay curves of the $2 \% \mathrm{Gd}$ concentration for $\mathrm{T} 2$ and $\mathrm{T} 2{ }^{*}$ sequences, respectively, using; the three shims (A, B), Five different pixel sizes $(\mathbf{C}, \mathbf{D})$, three slice thickness $(\mathbf{E}, \mathbf{F})$ and five TR values $(\mathbf{G}, \mathbf{H})$.

lowest magnitude measurements with low SD for the whole range of Gd concentrations. Measurements fluctuations can be observed at $4-20 \% \mathrm{Gd}$ concentrations and the magnitude values increased by nearly twice as much when using pixel sizes $3.28 \times 3.28 \mathrm{~mm}$ or $4 \times 4 \mathrm{~mm}$ compared to the $1 \times 1 \mathrm{~mm}$ pixel. $\mathrm{R}^{2}$ values ranged between 0.514 and 0.997 for all Gd concentrations using these pixel sizes, with the optimum obtained at $1 \times 1 \mathrm{~mm}$.

The slice thickness study on the $1 \%$ and $2 \%$ Gd using T2* sequence demonstrated higher magnitude measurements at $8 \mathrm{~mm}$, while the $12 \mathrm{~mm}$ thickness showed low $\mathrm{SD}$ for all the concentrations with $\mathrm{R}^{2}$ ranging $0.975-0.997$ at $\mathrm{p}<0.01$. Moreover, TR values had small effects on T2 measurements while $1-2 \%$ Gd concentration demonstrated a slight increase in measurements with TR. It was also observed that TR has a minor effect on $\mathrm{T} 2{ }^{\star}$ measurements with the lowest fluctuations of measurements found for concentrations of 3-20\% with $\mathrm{R}^{2}$ range between 0.98 and 0.996 . 


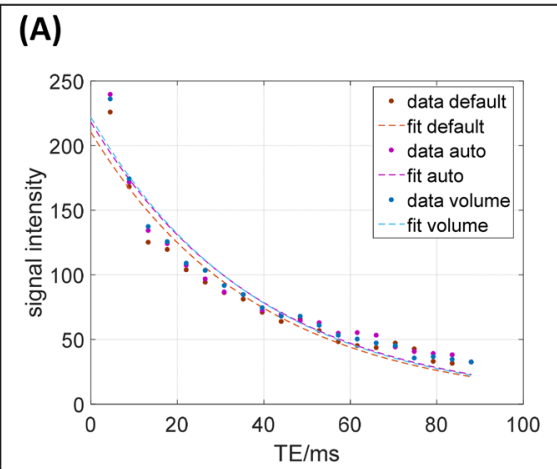

(C)

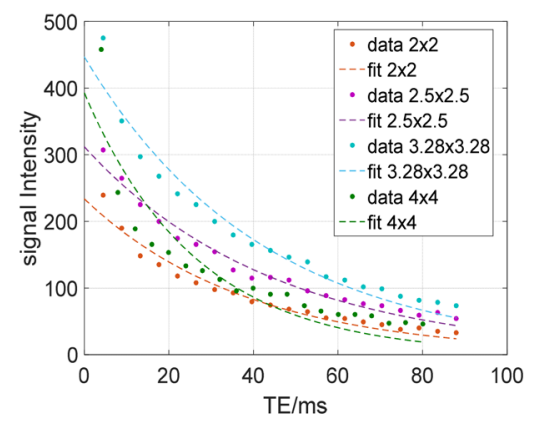

(E)

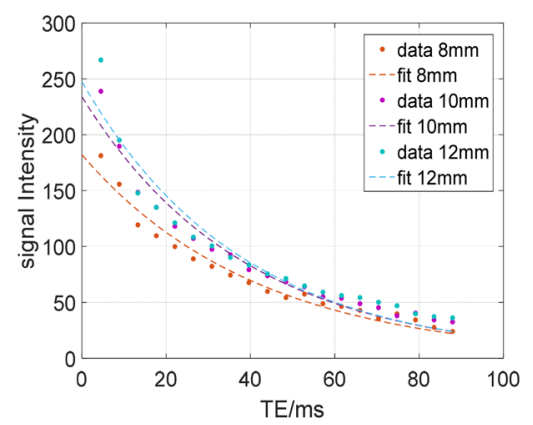

(G)

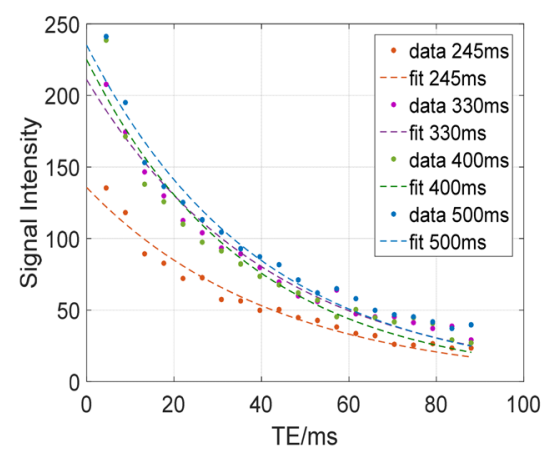

\section{(B)}

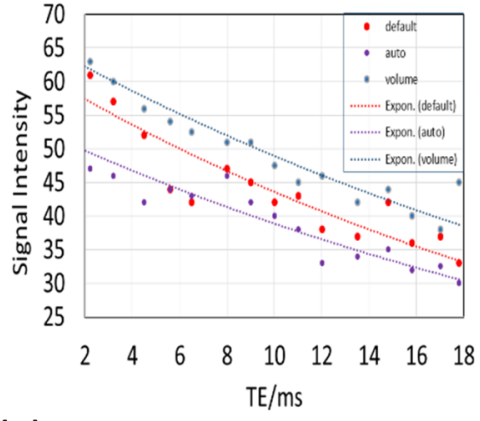

(D)

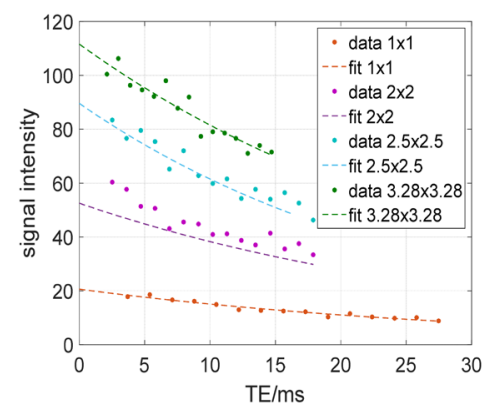

(F)

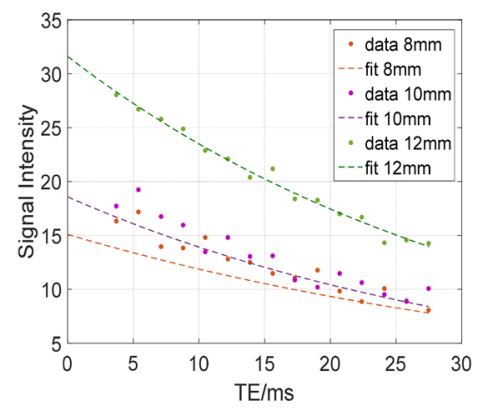

(H)

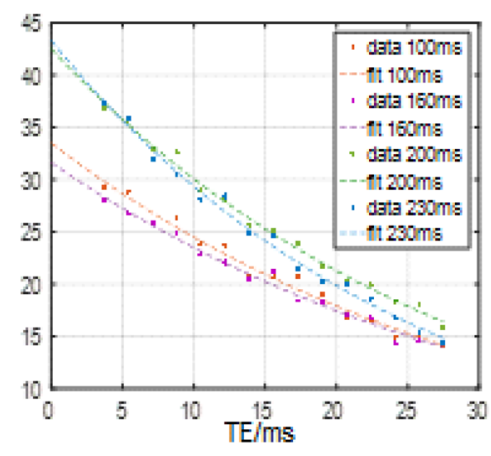

Figure 2. Decay curves of $\mathrm{T} 2$ and $\mathrm{T} 2{ }^{\star}$ for a healthy volunteer using: the three shim techniques (A, B), pixel sizes of $2 \times 2$ to $4 \times 4 \mathrm{~mm}$ for T2 (C), pixel sizes of $1 \times 1$ to $3.28 \times 3.28 \mathrm{~mm}$ for T2* $(\mathbf{D})$, slice thicknesses of 8,10 , $12 \mathrm{~mm}(\mathbf{E}, \mathbf{F})$, TR values $245-500 \mathrm{~ms}$ for T2 (G) and TR values $100-230 \mathrm{~ms}$ for T2* $(\mathbf{H})$.

Tables 5 and 6 show mean \pm SD of the result of magnitude measurements calculated on volunteers for both $\mathrm{T} 2$ and T2* sequences. Average T2 measurements on normal volunteers' livers using the T2 sequence in this study was $44 \pm 7 \mathrm{~ms}$ comparable with other similar studies ${ }^{31-33}$. We also observed that the average magnitude 


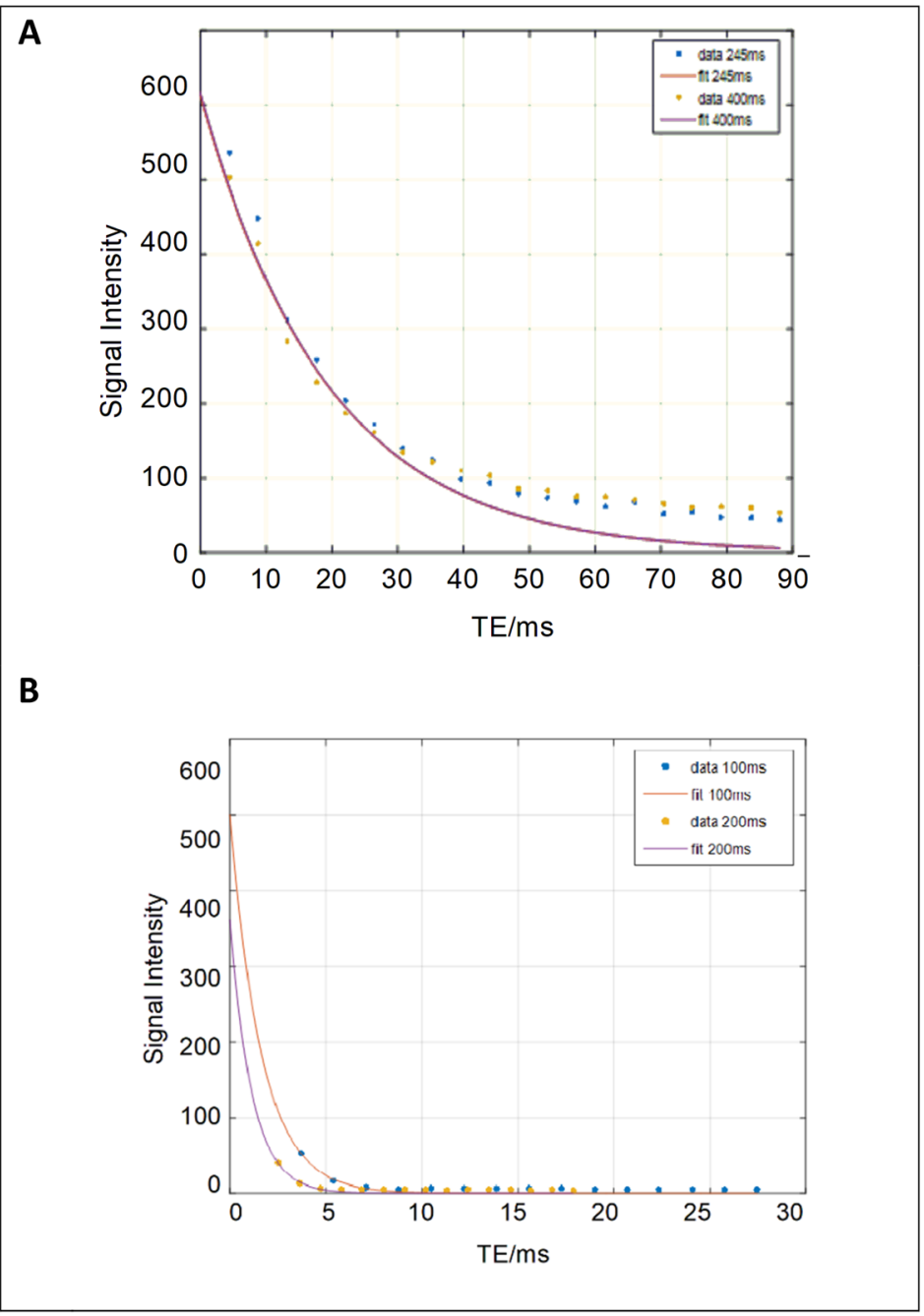

Figure 3. Decay curve of a sickle cell patient: (A) using auto shim, $2 \times 2 \mathrm{~mm}$ pixel size, $10 \mathrm{~mm}$ slice thickness, and $\mathrm{TR}=245 \mathrm{~ms}$ for $\mathrm{T} 2$ sequence ; $(\mathbf{B})$ at default shim, $1 \times 1 \mathrm{~mm}$ pixel size, $12 \mathrm{~mm}$ slice thickness, and $\mathrm{TR}=100 \mathrm{~ms}$ for $\mathrm{T}^{*}$ sequence.

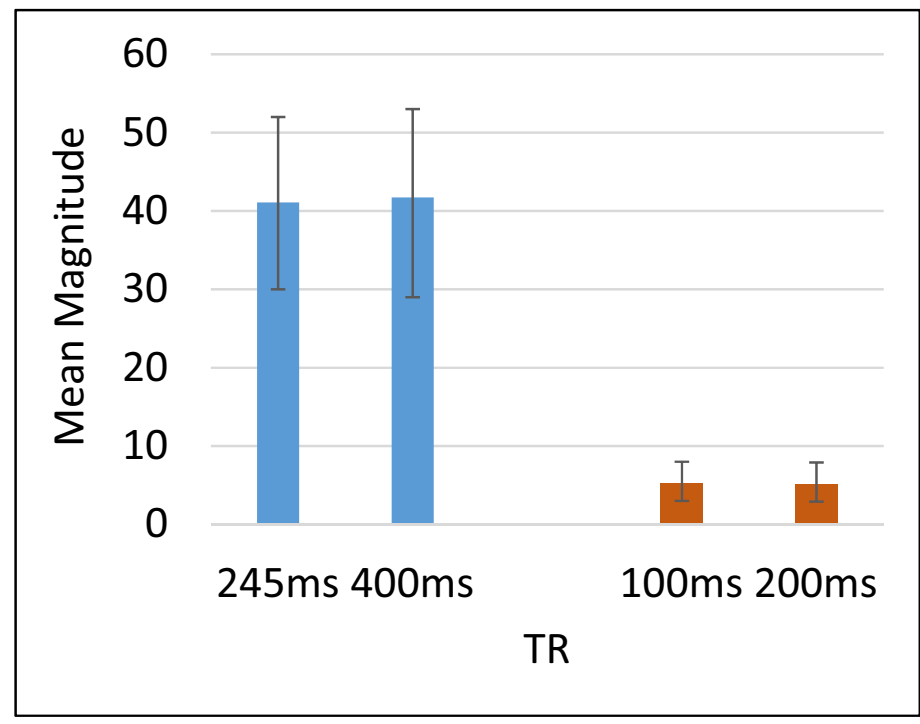

Figure 4. Mean magnitude measurements (with SD) of the 25 patients using the conventional $\mathrm{T} 2$ and $\mathrm{T} 2{ }^{*}$ sequences versus the new optimised sequences. 
A

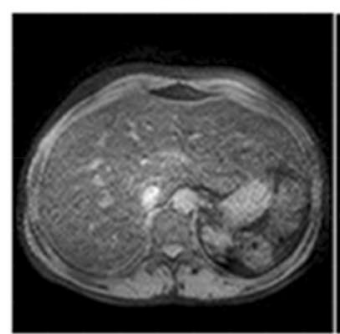

B

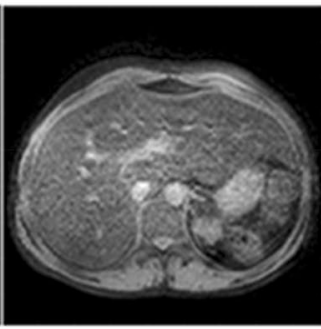

C

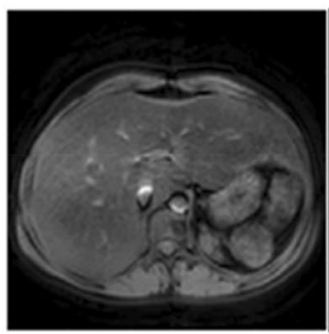

D

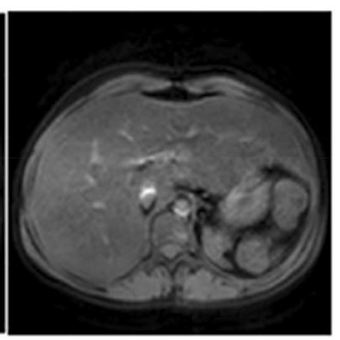

Figure 5. MRI images of the same patient using: (A) standard $\mathrm{T} 2^{\star},(\mathbf{B})$ optimised $\mathrm{T} 2{ }^{\star},(\mathbf{C})$ standard $\mathrm{T} 2$ and $(\mathbf{D})$ optimised $\mathrm{T} 2$ sequences.

\begin{tabular}{|l|l|l|l|l|l|l|l|}
\hline Sequence & Shim type & $\mathbf{1} \%$ & $\mathbf{2 \%}$ & $\mathbf{3} \%$ & $\mathbf{4 \%}$ & $\mathbf{5 \%}$ & $\mathbf{2 0 \%}$ \\
\hline \multirow{3}{*}{ T2 } & Default & $49.36 \pm 0.79$ & $26.01 \pm 0.33$ & $17.43 \pm 0.20$ & $13.70 \pm 0.15$ & $11.27 \pm 0.14$ & $5.58 \pm 1.49$ \\
\cline { 2 - 8 } & Auto & $49.57 \pm 0.14$ & $26.19 \pm 0.11$ & $17.52 \pm 0.17$ & $13.78 \pm 0.13$ & $11.29 \pm 0.13$ & $5.66 \pm 0.97$ \\
\cline { 2 - 8 } & Volume & $49.58 \pm 0.46$ & $25.91 \pm 0.58$ & $17.54 \pm 0.26$ & $13.75 \pm 0.21$ & $11.21 \pm 0.20$ & $6.06 \pm 1.27$ \\
\hline \multirow{3}{*}{ T2 $^{*}$} & Default & $34.90 \pm 2.81$ & $20.04 \pm 0.25$ & $14.07 \pm 0.33$ & $11.21 \pm 0.39$ & $8.08 \pm 0.29$ & $1.80 \pm 0.09$ \\
\cline { 2 - 8 } & Auto & $28.62 \pm 2.83$ & $19.21 \pm 0.22$ & $13.65 \pm 0.32$ & $11.02 \pm 0.23$ & $7.80 \pm 0.26$ & $1.70 \pm 0.09$ \\
\cline { 2 - 8 } & Volume & $30.62 \pm 3.45$ & $19.28 \pm 0.24$ & $13.88 \pm 0.33$ & $11.07 \pm 0.37$ & $7.83 \pm 0.26$ & $1.72 \pm 0.09$ \\
\hline
\end{tabular}

Table 1. Phantom mean measurements \pm standard deviation of $\mathrm{T} 2$ and $\mathrm{T} 2 *$ for different shim techniques.

\begin{tabular}{|l|l|l|l|l|l|l|l|}
\hline Sequence & Voxel size & $\mathbf{1} \%$ & $\mathbf{2} \%$ & $\mathbf{3} \%$ & $\mathbf{4}$ & $\mathbf{5 \%}$ & $\mathbf{2 0 \%}$ \\
\hline \multirow{5}{*}{ T2 } & $1 \times 1$ & $47.25 \pm 0.09$ & $24.30 \pm 0.1$ & $16.33 \pm 0.14$ & $12.61 \pm 0.10$ & $10.11 \pm 0.141$ & $4.79 \pm 0.80$ \\
\cline { 2 - 8 } & $2 \times 2$ & $50.57 \pm 0.14$ & $26.19 \pm 0.11$ & $17.52 \pm 0.17$ & $13.78 \pm 0.13$ & $11.29 \pm 0.132$ & $5.72 \pm 0.97$ \\
\cline { 2 - 8 } & $2.5 \times 2.5$ & $53.28 \pm 2.73$ & $27.66 \pm 0.32$ & $18.81 \pm 0.60$ & $14.38 \pm 0.46$ & $11.71 \pm 0.494$ & $6.00 \pm 0.99$ \\
\cline { 2 - 8 } & $3.28 \times 3.28$ & $54.23 \pm 3.64$ & $27.84 \pm 0.31$ & $19.17 \pm 0.79$ & $15.19 \pm 0.62$ & $12.86 \pm 0.589$ & $7.15 \pm 0.95$ \\
\cline { 2 - 8 } & $4 \times 4$ & $54.84 \pm 4.07$ & $27.99 \pm 0.39$ & $19.57 \pm 0.74$ & $15.88 \pm 0.60$ & $12.73 \pm 0.572$ & $7.29 \pm 1.00$ \\
\hline \multirow{5}{*}{ T2* } & $1 \times 1$ & $36.74 \pm 0.91$ & $21.18 \pm 0.31$ & $15.25 \pm 0.06$ & $12.01 \pm 0.20$ & $9.60 \pm 0.16$ & $1.75 \pm 0.12$ \\
\cline { 2 - 8 } & $2 \times 2$ & $44.10 \pm 1.76$ & $23.72 \pm 0.69$ & $18.52 \pm 0.29$ & $13.70 \pm 0.36$ & $10.28 \pm 0.38$ & $2.23 \pm 0.12$ \\
\cline { 2 - 8 } & $2.5 \times 2.5$ & $45.88 \pm 3.51$ & $24.21 \pm 0.36$ & $19.99 \pm 0.36$ & $13.89 \pm 0.34$ & $11.12 \pm 0.48$ & $2.01 \pm 0.12$ \\
\cline { 2 - 8 } & $3.28 \times 3.28$ & $50.72 \pm 7.78$ & $22.47 \pm 0.50$ & $20.13 \pm 0.40$ & $13.99 \pm 0.45$ & $11.16 \pm 0.49$ & $2.08 \pm 0.20$ \\
\cline { 2 - 8 } & $4 \times 4$ & $50.03 \pm 11.16$ & $20.82 \pm 1.54$ & $20.22 \pm 0.52$ & $14.00 \pm 0.44$ & $11.56 \pm 0.53$ & $2.21 \pm 0.37$ \\
\hline
\end{tabular}

Table 2. Phantom mean measurements \pm standard deviation for different pixel size using $\mathrm{T} 2$ and $\mathrm{T} 22^{\star}$.

\begin{tabular}{|l|l|l|l|l|l|l|l|}
\hline Sequence & Slice thickness $(\mathbf{m m})$ & $\mathbf{1} \%$ & $\mathbf{2 \%}$ & $\mathbf{3}$ & $\mathbf{4 \%}$ & $\mathbf{5 \%}$ & $\mathbf{2 0 \%}$ \\
\hline \multirow{3}{*}{$\mathrm{T} 2$} & 8 & $49.96 \pm 0.42$ & $27.24 \pm 0.17$ & $17.32 \pm 0.49$ & $13.01 \pm 0.26$ & $11.12 \pm 0.15$ & $5.40 \pm 0.79$ \\
\cline { 2 - 8 } & 10 & $52.57 \pm 0.09$ & $27.30 \pm 0.17$ & $17.47 \pm 0.44$ & $13.61 \pm 0.20$ & $11.18 \pm 0.14$ & $5.22 \pm 0.70$ \\
\cline { 2 - 8 } & 12 & $52.97 \pm 0.26$ & $27.19 \pm 0.17$ & $17.40 \pm 0.45$ & $13.67 \pm 0.21$ & $11.20 \pm 0.15$ & $4.88 \pm 0.71$ \\
\hline \multirow{3}{*}{$\mathrm{T}^{*}$} & 8 & $41.51 \pm 0.70$ & $24.05 \pm 0.25$ & $15.27 \pm 0.18$ & $12.36 \pm 0.22$ & $9.68 \pm 0.04$ & $2.42 \pm 0.06$ \\
\cline { 2 - 8 } & 10 & $36.74 \pm 0.51$ & $22.18 \pm 0.31$ & $15.25 \pm 0.06$ & $12.01 \pm 0.20$ & $9.60 \pm 0.03$ & $1.77 \pm 0.12$ \\
\cline { 2 - 8 } & 12 & $31.63 \pm 0.44$ & $22.13 \pm 0.30$ & $15.24 \pm 0.06$ & $12.02 \pm 0.05$ & $9.36 \pm 0.02$ & $1.75 \pm 0.01$ \\
\hline
\end{tabular}

Table 3. Phantom mean measurements \pm standard deviation of different slice thickness for $\mathrm{T} 2$ and $\mathrm{T} 2{ }^{*}$.

measurement using the default shim was less than the measurements recorded with auto and volume shim techniques by approximately 0.1 and $0.2 \%$, respectively. The default shim recorded high SD values, while the low fluctuation of measurements was recorded using the auto-shim technique. The overall T2* measurements recorded from the volunteers fell between 30 and $45 \mathrm{~ms}$ (average $36 \pm 5 \mathrm{~ms}$ ) comparable with literature ${ }^{34}$. The T2 magnitude measurements were presented with a slight increase for pixel sizes larger than $2.5 \times 2.5 \mathrm{~mm}$. 


\begin{tabular}{|l|l|l|l|l|l|l|l|}
\hline Sequence & TR & $\mathbf{1} \%$ & $\mathbf{2}$ & $\mathbf{3} \%$ & $\mathbf{4} \%$ & $\mathbf{5} \%$ & $\mathbf{2 0} \%$ \\
\hline \multirow{4}{*}{ T2 } & TR 212 & $49.47 \pm 0.48$ & $25.32 \pm 0.11$ & $16.56 \pm 0.22$ & $12.78 \pm 0.07$ & $10.06 \pm 0.13$ & $5.99 \pm 0.33$ \\
\cline { 2 - 8 } & TR 245 & $49.83 \pm 0.55$ & $25.51 \pm 0.11$ & $16.72 \pm 0.22$ & $12.84 \pm 0.06$ & $10.10 \pm 0.12$ & $4.83 \pm 0.40$ \\
\cline { 2 - 8 } & TR 330 & $49.88 \pm 0.23$ & $25.73 \pm 0.35$ & $17.07 \pm 0.31$ & $13.06 \pm 0.23$ & $10.10 \pm 0.13$ & $3.99 \pm 0.58$ \\
\cline { 2 - 8 } & TR 400 & $50.09 \pm 0.19$ & $26.50 \pm 0.40$ & $17.49 \pm 0.32$ & $13.50 \pm 0.20$ & $10.84 \pm 0.14$ & $4.72 \pm 0.87$ \\
\cline { 2 - 8 } & TR 500 & $50.15 \pm 0.19$ & $26.67 \pm 0.40$ & $17.66 \pm 0.34$ & $13.51 \pm 0.29$ & $10.84 \pm 0.15$ & $3.91 \pm 1.41$ \\
\hline \multirow{4}{*}{$2^{*}$} & TR 100 & $40.52 \pm 0.22$ & $19.45 \pm 0.12$ & $13.05 \pm 0.10$ & $9.82 \pm 0.04$ & $8.26 \pm 0.04$ & $1.61 \pm 0.09$ \\
\cline { 2 - 8 } & TR 160 & $40.66 \pm 0.28$ & $19.69 \pm 0.14$ & $13.07 \pm 0.18$ & $9.72 \pm 0.11$ & $8.19 \pm 0.05$ & $1.66 \pm 0.09$ \\
\cline { 2 - 8 } & TR200 & $40.68 \pm 0.34$ & $19.73 \pm 0.10$ & $13.24 \pm 0.06$ & $9.72 \pm 0.05$ & $8.36 \pm 0.02$ & $1.75 \pm 0.01$ \\
\cline { 2 - 8 } & TR 230 & $40.72 \pm 0.22$ & $19.66 \pm 0.19$ & $13.38 \pm 0.12$ & $9.78 \pm 0.11$ & $8.13 \pm 0.04$ & $1.63 \pm 0.13$ \\
\cline { 2 - 8 } & TR 300 & $40.86 \pm 0.34$ & $19.70 \pm 0.25$ & $13.31 \pm 0.13$ & $9.74 \pm 0.11$ & $8.15 \pm 0.12$ & $1.82 \pm 0.11$ \\
\hline
\end{tabular}

Table 4. Phantom mean measurements \pm standard deviation of different $\mathrm{TR}$ using $\mathrm{T} 2$ and $\mathrm{T} 2{ }^{\star}$ sequences.

\begin{tabular}{|c|c|c|}
\hline & T2 & T2* \\
\hline \multicolumn{3}{|l|}{ Shim } \\
\hline Default & $44.09 \pm 7.09$ & $36.87 \pm 11.93$ \\
\hline Auto & $44.73 \pm 6.25$ & $35.48 \pm 12.36$ \\
\hline Volume & $45.12 \pm 6.76$ & $36.37 \pm 12.17$ \\
\hline \multicolumn{3}{|l|}{ Pixel sizes } \\
\hline $1 \times 1$ & - & $34.10 \pm 7.71$ \\
\hline $2 \times 2$ & $44.09 \pm 6.07$ & $36.87 \pm 11.60$ \\
\hline $2.5 \times 2.5$ & $46.86 \pm 9.29$ & $37.15 \pm 12.93$ \\
\hline $3.28 \times 3.28$ & $47.60 \pm 9.66$ & $39.01 \pm 17.87$ \\
\hline $4 \times 4$ & $47.63 \pm 9.86$ & - \\
\hline \multicolumn{3}{|c|}{ Thicknesses } \\
\hline $8 \mathrm{~mm}$ & $43.33 \pm 7.26$ & $34.92 \pm 11.48$ \\
\hline $10 \mathrm{~mm}$ & $44.09 \pm 6.07$ & $34.54 \pm 7.71$ \\
\hline $12 \mathrm{~mm}$ & $44.09 \pm 6.45$ & $36.41 \pm 5.91$ \\
\hline
\end{tabular}

Table 5. Volunteers measurement of magnitude \pm standard deviation for $\mathrm{T} 2$ and $\mathrm{T} 2{ }^{\star}$ sequences.

\begin{tabular}{|l|l|l|l|}
\hline TR & T2 & TR & T2 $^{*}$ \\
\hline TR245 & $45.65 \pm 6.62$ & TR100 & $33.84 \pm 7.44$ \\
\hline TR330 & $46.66 \pm 6.41$ & TR160 & $34.14 \pm 7.20$ \\
\hline TR400 & $46.09 \pm 6.52$ & TR200 & $36.41 \pm 5.91$ \\
\hline TR500 & $47.39 \pm 4.95$ & TR230 & $36.58 \pm 5.84$ \\
\hline
\end{tabular}

Table 6. Volunteers measurement of magnitude \pm standard deviation of the ten volunteers using different TR values for both $\mathrm{T} 2$ and $\mathrm{T} 2{ }^{\star}$ sequences.

The $\mathrm{T} 2{ }^{*}$ magnitude measurements at different pixel sizes showed similarity to the magnitude measurement obtained for the $1 \% \mathrm{Gd}$ concentration. The lowest measurement was recorded at $1 \times 1 \mathrm{~mm}$. Larger pixel sizes provided $\mathrm{T}^{\star}$ measurements of up-to $20 \%$ than that of the $1 \times 1 \mathrm{~mm}$. It is also observed that the SD values became higher with pixel size (reaching up to $50 \%$ for pixel size of $4 \times 4 \mathrm{~mm}$ ).

In comparison with the literature, our study found that the increase of pixel size resulted in an increase in the sensitivity of the magnitude measurements ${ }^{35}$. Larger pixel sizes have caused the measurements to become less reliable. Similar to the T2 measurements the utilization of higher receiver bandwidth in this study incorporated more noise on the acquired images, and subsequently caused scattering in signal intensity measurements. This led to wide fluctuations of measurements especially in the case of high pixel sizes of $3.28 \times 3.28 \mathrm{~mm}$ and $4 \times 4 \mathrm{~mm}$ $\left(\mathrm{R}^{2} \sim 0.614\right)$. The T2 sequence results showed no significant differences between the magnitude measurements for the slice thicknesses of 8,10 , and $12 \mathrm{~mm}$. As for T2*, the $12 \mathrm{~mm}$ thickness was associated with the highest magnitude measurements and lowest fluctuations of the measurements.

Mean magnitude measurements of T2 for the range of studied TR witnessed no significant difference in their values. T2* measurements at TR values of 100-160 ms, recorded low mean magnitude of measurements (by $30 \%$ ) compare to those at 200-230 ms. Long T2* components, like those found in healthy volunteers, require 
long TR values for accurate measurements. It is observed that long TRs of 200-230 ms provides consistent results ( $\mathrm{R}^{2}$ range $\left.0.95-0.99\right)$.

In general, there was a slight difference in magnitude measurements between the conventional and optimised T2 sequences for patients studies (Figs. 3, 4). The conventional sequence provided a higher measurement by $1.5 \%$ and higher SD by $4 \%$ than its corresponding optimised sequence. The correlation coefficients obtained from the conventional sequence were 0.978-0.994, whilst for the optimised sequence the range was 0.979-0.994 $(\mathrm{p}<0.01)$. We also observed similar image qualities were obtained for the patients using the T2 and T2* optimised sequences as compared to their corresponding standard sequences (Fig. 5).

Thus, the results indicate that the standard T2 sequence could easily replace the optimised sequence without compromising sickle cell patients' measured data with iron overload. Similarly results obtained for T2* sequences were in close agreement with this conclusion (with uncertainty of 3\%). The correlation coefficient for the standard sequence ranged between $0.942-0.99$, while for the optimised $(0.945-1.0)$ at $p$-value $<0.01$.

Our studies on the effects of these parameters were for the first time investigated on the livers of transfusiondependent paediatric patients using both $\mathrm{T} 2$ and $\mathrm{T} 2{ }^{\star}$ sequence. Our investigations showed a room for further improvement in the $\mathrm{T} 2^{\star}$ sequence by adopting the optimised sequence instead of the conventional sequence. Another advantage of this optimisation is that the acquisition time for the T2 sequence was reduced by almost $38 \%$ and for $\mathrm{T} 2 *$ by $25 \%$. We also noticed that the measurements' reliability has dramatically improved.

This study has some limitations to generalize its outcomes for a number of reasons: (i) the number of samples is relatively small (10 volunteers +25 patients). (ii) To get the optimum pixel size and keep the Hz/pixel fixed, this requires a change in both the field of view (FOV) and the matrix size. This type of modification affects the routine of work in the hospital so we had to keep the FOV and the size of the matrix constant throughout the study period.

\section{Conclusion}

The aim of this study was to investigate the effect of different shim techniques, voxel sizes, and repetition time (TR) on $\mathrm{T} 2$ and $\mathrm{T} 2{ }^{*}$ sequences and to determine optimum settings for the evaluation of the amount of iron in the livers of transfusion dependent sickle cell patients. Phantom with different concentrations of Gd (1-20\%), and ten volunteers were used to define these settings, which were then investigated on 25 patients. The optimized protocols resulted in a reduction of acquisition time by $38 \%$ and $25 \%$ for $\mathrm{T} 2$ and $\mathrm{T} 2{ }^{*}$, respectively. In addition, the quality of images were not compromised as compared to the conventional sequences. The outcomes of this study could be particularly beneficial for young adults who cannot tolerate MRI scans for long periods of time. The research results indicated that $\mathrm{T} 2$ and $\mathrm{T} 2{ }^{\star}$ sequences can be further refined to provide more reliable measurements and on transfusion dependent-sickle cell patients.

Received: 11 October 2020; Accepted: 7 April 2021

Published online: 19 April 2021

\section{References}

1. Marotta, C. A., Wilson, J. T., Forget, B. G. \& Weissman, S. M. Human 13-globin messenger RNA 111: nucleotide sequences derived from complementary DNA. J. Biol. Chem. 25, 5040-5053 (1977).

2. Kirkham, F. J. Therapy insight: stroke risk and its management in patients with sickle cell disease. Nat. Clin. Pract. Neurol. 3, 264-278 (2007).

3. Archibald, R. G. A case of sickle cell anemia in the Sudan. Tran. R. Soc. Trop. Med. Hyg. 19, 389-393 (1926).

4. Ahmed, H. A. \& Baker, E. A. Sickling in the Sudan; result of surveys in Blue Nile Province. East Afr. Med. J. 6, 395-399 (1986).

5. Daak, A. A. et al. Effect of Omega-3 (n-3) fatty acid supplementation in patients with sickle cell anemia: randomized, double-blind, placebo-controlled trail. Am. J. Clin. Nurt. 97, 37-44 (2013).

6. Makani, J. et al. Mortality in sickle anemia in Africa: a prospective cohort study in Tanzania. PLoS ONE 6, el4699 (2011).

7. Bayav, M., Ozbulbul, N. I. \& Bor, O. Assessment of cardiac and liver iron overload by magnetic. J. Int. Med. Res. 48(7), 1-6 (2020).

8. Padeniya, P. et al. Comparison of liver MRI R2(FerriScan) VS liver MRI T2* as a measure of body iron load in a cohort of beta thalassaemia major patients. Orphanet. J. Rare Dis. 15, 26 (2020).

9. Henninger, B., Alustiza, J., Garbowski, M. \& Gandon, M. Practical guide on the quantification of hepatic iron with MRI. Eur. Radiol. 30, 383-439 (2020).

10. Wood, J. C. et al. Liver iron concentration measurements by MRI in chronically transfused children with Sickle Cell Anemia: baseline results from the TWiTCH Trail. Am. J. Hematol. 90, 806-810 (2015).

11. Zaman, A. et al. Robust myocardial T2 and T2* mapping at 3T. J. CMR 41, 1013-1020 (2015).

12. Hancu, I., Govenkar, A., Lenkinski, R. E. \& Lee, S. K. On shimming approaches in 3T breast MRI. MRM 69, 862-867 (2013).

13. Mulder, M. J., Keuken, M. C., Bazin, P. L., Alkemade, A. \& Forstmann, B. U. Size and shape matter: the impact of voxel geometry on the identification of small nuclei. PLoS ONE https://doi.org/10.1371/journal.pone.0215382 (2019).

14. Pavitt, H. L. et al. The effect of reducing repetition time TR on the measurement of liver R2 for the purpose of measuring liver iron concentration. MRM 65, 1346-1351 (2011).

15. Wood, J. C. et al. Cardiac iron determines cardiac $\mathrm{T} 22^{\star}, \mathrm{T} 2$ and $\mathrm{T} 1$ in the gerbil model of iron cardiomyopathy. Circulation 112(535), $543(2005)$

16. He, T. et al. Multi-center transferability of a breath-hold T2 technique for myocardial iron assessment. J. Cardiovasc. Magn. Reson. 10, 11 (2008).

17. Westwood, M. A. et al. Interscanner reproducibility of cardiovascular magnetic resonance $\mathrm{T} 2^{\star}$ measurements of tissue iron in thalassemia. J. Magn. Reson. Imaging 18, 616-620 (2003).

18. Snel, G. H. et al. Cardiovascular Magnetic Resonance native quantitative values for $\mathrm{T} 2{ }^{\star}$ and $\mathrm{T} 2$ cardiomypathies and heart transplantations: a systematic review and meta-analysis. JCMR 22, 44 (2020).

19. Messroghli, J. et al. Clinical recommendations for cardiovascular magnetic resonance mapping of $\mathrm{T} 1, \mathrm{~T} 2, \mathrm{~T} 22^{*}$ and extracellular volume: a consensus statement by the Society for Cardiovascular Magnetic Resonance (SCMR) endorsed by the European Association for Cardiovascular Imaging (EACVI). JCMR 19, 75 (2017).

20. Schwitter, J. Comparison of three different cardiac T2-mapping techniques at 1.5 tesla. Biomed. J. Sci. Tech. Res. 3(2), 3143-3150 (2018). 
21. Labranche, R. et al. Liver iron quantification with MR imaging: a primer for radiologists. Radiographics 38, 392-412 (2018).

22. Sammet, C. L. et al. Measurement and correction of stimulated echo contamination in T2-based iron quantification. Magn. Reson. Imaging 31, 664-668 (2013).

23. Rohrer, M., Bauer, H., Mintorovitch, J., Requardt, M. \& Weinmann, H. J. Comparison of magnetic properties of MRI contrast media solutions at different magnetic field strengths. Invest. Radiol. 40, 715-724 (2005).

24. Kanematsu, M. et al. Thick-section half-Fourier rapid acquisition with relaxation enhancement MR cholangiopancreatography: effects of iv administration of gadolinium chelate. AJR Am. J. Roentgenol. 178, 755-761 (2002).

25. Kuperman, V. Y. \& Alley, M. T. Differentiation between the effects of T1 and T2* shortening in contrast-enhanced MRI of the breast. J. Magn. Reson. Imaging 9, 172-176 (1999).

26. May, D. A. DJ Pennington (2000) Effect of gadolinium concentration on renal signal intensity: an in vitro study with a saline bag model. Radiology 216, 232-236 (2000).

27. Lee, M. J. et al. The T2-shortening effect of gadolinium and the optimal conditions for maximizing the CNR for evaluating the biliary system: a phantom study. Korean J. Radiol. 12(3), 364 (2011).

28. Donald, W. M. From Picture to Proton, 2nd edn, 2007, ISBN-13: 978-0521683845.

29. Alam, M. H. et al. Validation of T2* in-line analysis for tissue iron quantification. JCMR 18, 23 (2016).

30. Ghurge, N. R., Enriquez, C. M., Coates, D. T., Nelson, M. D. \& Wood, J. C. Improved R2* measurements in myocardial iron overload. J. Magn. Reson. Imaging 23, 9-16 (2006).

31. Abe, Y., Yamashita, Y., Tang, Y., Namimoto, T. \& Takahashi, M. Calculation of T2 relaxation time from ultrafast single shot sequences for differentiation of liver tumors: comparison of echo-planar, HASTE, and spin-echo sequences. Radiat. Med. 18, 7-14 (2000).

32. Foley, W. D. et al. Contrast optimization for the detection of focal hepatic lesions by MR imaging at 1.5 T. AJR Am. J. Roentgenol. 149, 1155-1160 (1987).

33. Bernardino, M. E., Chaloupka, J. C., Malko, J. A., Chezmar, J. L. \& Nelson, R. C. Are hepatic and muscle T2 values different at 0.5 and 1.5 Tesla?. Magn. Reson. Imaging 7, 363-367 (1989).

34. Obrzut, M. et al. Value of liver iron concentration in healthy volunteers assessed by MRI. Sci. Rep. 10, 17887 (2020).

35. Chavhan, G. B., Babyn, P. S., Thomas, B., Shroff, M. M. \& Haacke, E. M. Principles, techniques, and applications of T2*-based MR imaging and its special applications. Radiographics 29, 1433-1449 (2009).

\section{Acknowledgements}

The authors appreciate the help of Al Ateeba Hospital staff and specifically to Mr. Ahmed Abdelfatah for his time and dedication and for arranging the scanning time at their busy hospital. The authors gratefully acknowledge The Deanship of Scientific Research (DSR) at King Abdulaziz University, Jeddah DSR for technical and financial support under grant no. RG-12-135-41.

\section{Author contributions}

In this work, the principal investigator A.A. was encouraged by the research supervisor I.S. to investigate the possibility of employing magnetic resonance imaging technology for the required measurements in the research problem that was discussed in details with the I.S., A.A. made all arrangements for collecting data. With the assistance of both A.B. and H.M. all work were done, including lab, patient checks, and arranging times for sample collection in the hospital. The collected data was analysed by A.A. and I.S. and then A.A. prepared the draft that include writing the main text and drawing the figures and graphs, reviewed and edited by I.S. The final reviews were done by A.S. with assistance and support from F.A.

\section{Competing interests}

The authors declare no competing interests.

\section{Additional information}

Correspondence and requests for materials should be addressed to A.A. or I.S.

Reprints and permissions information is available at www.nature.com/reprints.

Publisher's note Springer Nature remains neutral with regard to jurisdictional claims in published maps and institutional affiliations.

(c) (i) Open Access This article is licensed under a Creative Commons Attribution 4.0 International (c) License, which permits use, sharing, adaptation, distribution and reproduction in any medium or format, as long as you give appropriate credit to the original author(s) and the source, provide a link to the Creative Commons licence, and indicate if changes were made. The images or other third party material in this article are included in the article's Creative Commons licence, unless indicated otherwise in a credit line to the material. If material is not included in the article's Creative Commons licence and your intended use is not permitted by statutory regulation or exceeds the permitted use, you will need to obtain permission directly from the copyright holder. To view a copy of this licence, visit http://creativecommons.org/licenses/by/4.0/.

(C) The Author(s) 2021, corrected publication 2021 